\title{
Hepatocellular carcinoma patients highly and specifically expressing XAGE-1 exhibit prolonged survival
}

\author{
LEI GONG, JIRUN PENG, ZHUQINGQING CUI, PENGCHENG CHEN, \\ HUI HAN, DAFANG ZHANG and XISHENG LENG
}

Department of Hepatobiliary Surgery, Peking University People's Hospital, Beijing, P.R. China

Received July 12, 2010; Accepted September 11, 2010

DOI: $10.3892 / \mathrm{ol} .2010 .175$

\begin{abstract}
XAGE-1 is classified into the group of a new family of cancer-testis antigens (CTA) and has the four transcript variants of XAGE-1a, XAGE-1b, XAGE-1c and XAGE-1d. Immunohistochemistry was used to investigate the expression of XAGE-1 transcript variants in Chinese patients with hepatocellular carcinoma (HCC). Reverse transcriptionpolymerase chain reaction (RT-PCR) and real-time RT-PCR were used to analyze XAGE-1 gene expression, and XAGE-1 protein expression was examined by immunohistochemistry. Furthermore, the clinical correlation of XAGE-1 expression was analyzed. The expression of the XAGE-1 mRNA was investigated in the tissues of $96 \mathrm{HCC}$ patients and all XAGE-1 isoforms were detected in these tissues. Three types of XAGE-1 transcript variants (XAGE-1b, XAGE-1c and XAGE-1d) showed high specific and frequent expression in HCC tissues, with the positive expression rate of XAGE-1b, XAGE-1c and XAGE-1d being 41.7\% (40/96), $15.6 \%(15 / 96)$ and $26.0 \%$ (25/96), respectively. XAGE-1b was the dominant type, but none of the three were detected in adjacent non-HCC tissues. Only 2 cases of XAGE-1a mRNA expression were observed. Moreover, XAGE-1 protein was detected in 39 of 96 HCC patients, but none in the adjacent non-cancerous tissue and normal liver tissue. No relationship was found between the expression of XAGE-1 and clinical parameters, such as age, gender, tumor size, TNM staging, serum AFP level and infection with hepatitis virus. Patients with XAGE-1b-positive transcript variant exhibited shorter 2-year survival times. The high frequency and specificity of XAGE-1, particularly XAGE-1b, in HCC indicates that their products may predict the prognosis of HCC patients and be novel targets for antigen-specific immunotherapy to HCC.
\end{abstract}

Correspondence to: Dr Xisheng Leng, Department of Hepatobiliary Surgery, Peking University People's Hospital, No. 11 South Xizhimen Street, Xicheng, Beijing 100044, P.R. China

E-mail: lengxs2003@126.com

Key words: hepatocellular carcinoma, XAGE-1, cancer/testis antigen

\section{Introduction}

Human hepatocellular carcinoma (HCC) is a common malignancy, with high incidence and mortality rates worldwide, particularly in East Asia (1). Hepatic surgery is the normal treatment and has undergone improvement; however, recurrence and metastasis rates remain high $(2,3)$. Therefore, it is crucial to identify and establish effective novel approaches to treat HCC. In the past two decades, immunotherapy has proven to be a treatment strategy, and active immunotherapy approaches using specific vaccines appear to have potential in the treatment of HCC patients.

In recent years, a new family of tumor-specific antigens has been identified, known as cancer/testis antigens (CTA). CTA is a family that includes various types of tumor-specific antigens primarily expressed in primitive germ cells, spermatogonia and certain human tumors. The malignant transformation of these antigens is frequently associated with the activation or depression of genes, resulting in the expression of the antigens. More than 50 types of CTA have been identified thus far, including MAGE, GAGE, PAGE, NY-ESO-1, SSX, SPANX and XAGE (4-8).

XAGE-1 was originally identified by database mining of expressed sequence tags and belongs to a new family of CTA with an expression pattern that is limited to the germ cells of the testis and a variety of neoplastic tissues with immunogenicity $(9,10)$, but is abundantly expressed in breast, prostate and lung cancer as well as in Ewing's sarcomas and rhabdomyosarcomas (11-14). The XAGE-1 gene lies on the $\mathrm{X}$ chromosome and its encoded proteins have strong homology with members of the GAGE/PAGE family in the $\mathrm{COOH}$ terminus. XAGE-1 expression in testis and cancer tissues is regulated by methylation of the $\mathrm{CpG}$ island in the gene promoter (15). There are 4 transcript variants of XAGE-1, i.e., XAGE-1a, XAGE-1b, XAGE-1c and XAGE-1d (10,13,16), with XAGE-1b and XAGE-1d being specifically expressed in lung and prostate cancer. These variants are immunogenic and can therefore be used as immunotherapy targets. However, only limited research haa been performed on XAGE-1 in HCC. The present study showed that XAGE-1b was expressed strongly with a high frequency in HCC, while XAGE-1d relatively less strongly with a lower frequency. Additionally, more information is provided on this cancer antigen family and its potential use in the diagnosis of or immunotherapy for HCC. 


\section{Materials and methods}

Patients and specimens. A total of 96 HCCs and corresponding adjacent non-cancerous liver tissues from patients at Peking University People's Hospital, China, were surgically resected and dissected into sections of $\sim 0.3 \times 0.3 \times 0.3 \mathrm{~cm}$ and immediately stored in liquid nitrogen. Written informed consent was obtained from each patient for the use of the specimens and their clinicopathological characteristics. The study was approved by the Ethics Committee of Peking University People's Hospital. HCC diagnosis was confirmed by pathological examination and the TNM stage was determined on the basis of the criteria issued by UICC in 2002 .

Total RNA isolation and cDNA synthesis. Total RNA was isolated from the frozen tumors and adjacent non-cancerous liver specimens using RNeasy mini kit (Qiagen, Hilden, Germany). A total of $2 \mu \mathrm{g}$ was reverse-transcribed into cDNA using M-MLV reverse transcriptase (Promega, Madison, WI, USA). In addition to 200 units M-MLV, the reaction mixture consisted of $1 \mu 1$ oligo (dT) $)_{15}$ as a primer (Promega), 0.5 units RNase ribonuclease inhibitor (Promega), $4 \mu \mathrm{l}$ of RT buffer (250 mM Tris-HCl, pH 8.3, $375 \mathrm{mM} \mathrm{KCl,} 15 \mathrm{mM} \mathrm{MgCl}_{2}$ and $50 \mathrm{mM}$ DTT) and $1 \mu \mathrm{l}$ of $10 \mathrm{mM}$ dNTPs (Phamcia), with DEPC-treated water added to a final volume of $20 \mu 1$. The mixture was incubated for $5 \mathrm{~min}$ at $70^{\circ} \mathrm{C}$ and $5 \mathrm{~min}$ on ice, $60 \mathrm{~min}$ at $42^{\circ} \mathrm{C}$ and $5 \mathrm{~min}$ at $95^{\circ} \mathrm{C}$. The cDNAs were tested for integrity by amplifying the GAPDH gene.

Reverse transcription-polymerase chain reaction (RT-PCR) for XAGE-1 gene. A schematic representation of the structure of the XAGE-1 gene and transcripts, including locations of primers used in this study, is shown in Fig. 1. Primer pair sequences used for RT-PCR are listed in Table I, with X-7 and X-2 for XAGE-1a, X-1 and X-2 for the common region of XAGE- $1 b, X-4$ and X-2 for XAGE-1c and X-6 and X-2 for XAGE-1d. The amplification program for XAGE-1 transcript variants was $1 \mathrm{~min}$ at $94^{\circ} \mathrm{C}, 1 \mathrm{~min}$ at $60^{\circ} \mathrm{C}$ and $1 \mathrm{~min}$ at $72^{\circ} \mathrm{C}$ for 30 cycles after $10 \mathrm{~min}$ at $94^{\circ} \mathrm{C}$. The amplification program for GAPDH was $30 \mathrm{sec}$ at $94^{\circ} \mathrm{C}, 45 \mathrm{sec}$ at $57^{\circ} \mathrm{C}$ and $45 \mathrm{sec}$ at $72^{\circ} \mathrm{C}$ for 30 cycles after $10 \mathrm{~min}$ at $94^{\circ} \mathrm{C}$. The cycles were followed by a 10 -min elongation step at $72^{\circ} \mathrm{C}$. The PCR products were analyzed on $1.6 \%$ agarose gel electrophoresis. The positive PCR products were then recovered and sequenced. Data were analyzed by comparison with the respective sequences in GeneBank.

Real-time reverse transcription-polymerase chain reaction (RT-PCR) for GAPDH and XAGE-1. Sequences of genespecific primers for XAGE-1b and XAGE-1d GAPDH are shown in Table I. Relative quantification using real-time RT-PCR was performed using SYBR-Green PCR Mastermix (Applied Biosystems, Foster City, CA, USA) in accordance with the manufacturer's instructions, and the ABI PRISM 7000 Sequence Detection System (Applied Biosystems). Amplification was performed for 42 cycles with $1 \mu \mathrm{l}$ cDNA (corresponding to $60 \mathrm{ng}$ total RNA) solution extracted from tumor and non-cancerous specimens with $10 \mu \mathrm{l}$ PCR SYBRGreen Master Mix (Applied Biosystems) and $1 \mu 1750 \mathrm{nM}$ forward and reverse primers in a total volume of $20 \mu \mathrm{l}$. The

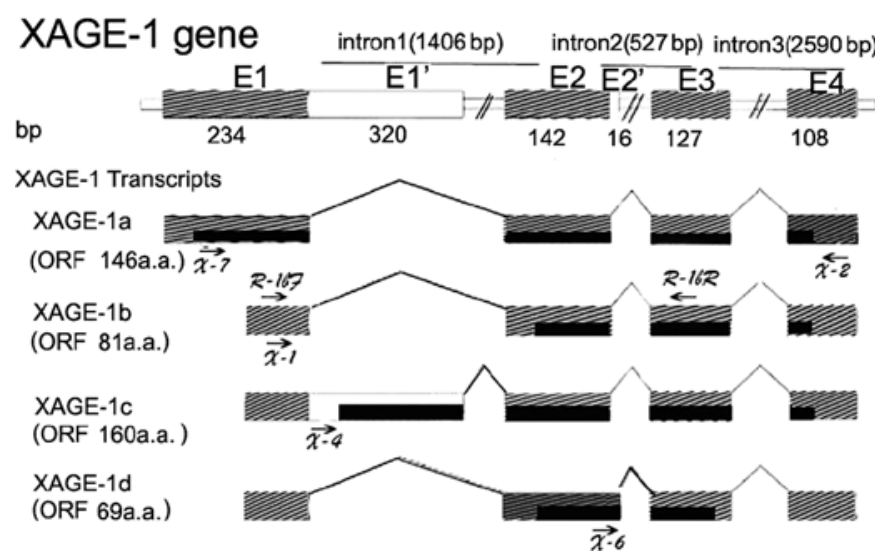

Figure 1. Schematic representation of the XAGE-1 gene and its transcripts and open reading frames (ORFs). Exons are shown as black boxes and introns as white boxes. ORFs are shown in hatched boxes. Arrows indicate the primers used in our study.

amplification conditions were: $95^{\circ} \mathrm{C}$ for $15 \mathrm{sec}$ and $60^{\circ} \mathrm{C}$ for $60 \mathrm{sec}$.

Immunohistochemistry. Paraffin-embedded tumors and the adjacent non-cancerous tissues of $4 \mu \mathrm{m}$ were mounted on glass slides and deparaffinized with xylene and ethanol. For antigen retrieval, the tissues were water-bath heated in an antigen retrieval buffer [10 $\mathrm{nM}$ citrate buffer $(\mathrm{pH}$ 6.0)] for $10 \mathrm{~min}$. Endogenous peroxidase was inactivated with $0.3 \% \mathrm{H}_{2} \mathrm{O}_{2}$ for $10 \mathrm{~min}$. Following pre-incubation with serumfree blocking solution, XAGE-1 mAb (Santa Cruz) was added at a concentration of $2 \mu \mathrm{g} / \mathrm{ml}$ and incubated at $4^{\circ} \mathrm{C}$ overnight. Following PBS washing, biotin-conjugated goat anti-mouse IgG and horseradish peroxidase-conjugated avidin (Santa Cruz) were applied, respectively, and incubated for $30 \mathrm{~min}$ at room temperature for each step. The specimens were then visualized using 3,3'-diaminobenzidine (DAB) in $\mathrm{H}_{2} \mathrm{O}_{2}$ and counterstained with hematoxylin solution.

Statistical analysis. Associations between the XAGE-1 expression and the clinicopathological characteristics were analyzed using the Chi-square or Fisher's exact test, according to the test condition. The survival probabilities were estimated using the Kaplan-Meier method and were compared using the log-rank test. Overall survival time was defined as the time from the date of surgery to the date the patient succumbed to any cause. Patients who were alive at the date of the last follow-up were censored on that date plus 1 day. Confidence intervals of $95 \%$ were used throughout the analysis. Statistical significance was defined as $\mathrm{P}<0.05$. The statistical tests were performed using the Statistical Package, SPSS 13.0 for Windows (SPS Inc., Chicago, IL, USA).

\section{Results}

XAGE-1 mRNA expression in hepatocellular carcinoma. Expression of the four XAGE-1 transcript variants was investigated in $96 \mathrm{HCC}$ and adjacent non-cancerous liver tissues by conventional 30-cycle RT-PCR using specific primer pairs (Fig. 1, Table I). The PCR product was analyzed in $1.6 \%$ agarose gel and sequenced. Fig. 2 shows a representative 
Table I. PCR primers used in this study, including GAPDH and XAGE-1.

\begin{tabular}{lrr}
\hline Primer & Sequence & Notes \\
\hline GAPDHF & 5'-GAAGGTGAAGGTCGGAGTC-3' & Forward, sense \\
GAPDHR & 5'-GAAGATGGTGATGGGATTTC-3' & Reverse, anti-sense \\
X-7 & 5'-ACCTCAGTGCGCATGTTCAC-3' & Forward, sense \\
X-1 & 5'-TTTCTCCGCTACTGAGACAC-3' & Forward, sense \\
X-4 & 5'-CTGGGAGTTGAAGTGTGAGT-3' & Forward, sense \\
X-2 & 5'-CAGGTGCTGGGAAGGGAAAT-3' & Forward, sense \\
X-6 & 5'-CAGCTTGCGTTGTTTCAGCT-3' & Reverse, anti-sense \\
R-1bF & 5'-TACTGAGACACGGCGGAC-3' & Forward, sense \\
R-1bR & 5'-TTCCATGTCGCGCACTG-3' & Reverse, anti-sense
\end{tabular}

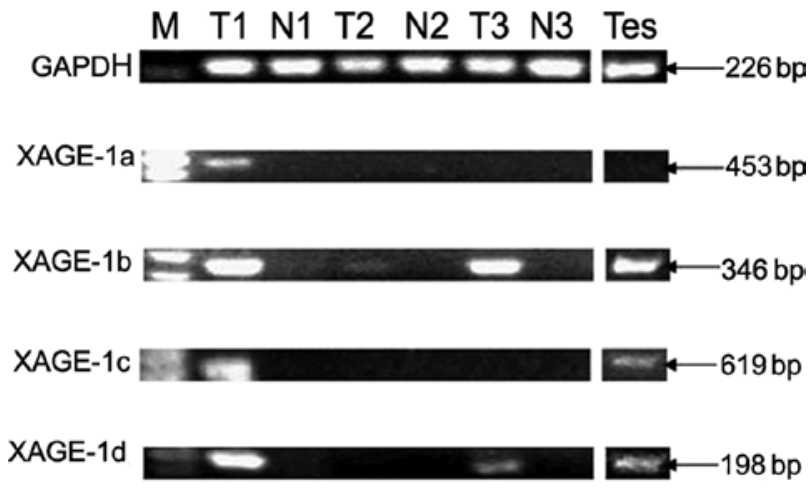

Figure 2. XAGE-1 mRNA expression in hepatocelluar carcinoma, as well as adjacent non-cancerous and testis tissues. GAPDH was used for the internal controls. XAGE-1b was confirmed by the negative expression of XAGE-1a and nucleotide sequence. M, 50-bp marker; T, tumor; N, adjacent tissue and Tes, testis.

RT-PCR and Table II summarizes the results. The expression of XAGE-1b, XAGE-1d and XAGE-1c mRNA, but not XAGE-1a, was observed, with the exception of 2 cases. Histologically, XAGE-1b mRNA expression was observed in 40 of $96(41.7 \%)$ HCC patients. By contrast, XAGE-1d mRNA expression was observed in $25(15.6 \%) \mathrm{HCC}$ specimens and XAGE-1c mRNA in $15(26.0 \%)$ HCC specimens. XAGE-1a mRNA expression was observed in only 2 cases of HCC tissues. XAGE-1c and XAGE-1d mRNA expression, except for 1 case, was associated with XAGE-1b mRNA expression. No XAGE-1 mRNA expression was observed in the 96 adjacent normal liver tissues.

Quantitative real-time $R T-P C R$ analysis of XAGE-1b and $X A G E-1 d$ mRNA expression in HCC, adjacent normal liver tissues and testis tissues. XAGE-1b and XAGE-1d mRNA expression was quantitatively analyzed by real-time RT-PCR using SYBR-Green PCR Mastermix with the procedure running on the ABI PRISM 7000 Sequence Detection System and specific primers shown in Fig. 1 and Table I. As shown in Fig. 3, compared to GAPDH expression, the XAGE-1b and XAGE-1d mRNA copy numbers ranged from 0.02 to $74.74 \%$ and 0.001 to $44.44 \%$, respectively. Additionally, the mean values were 9.73 and $4.81 \%$, respectively, and standard deviation was 14.48 and $9.85 \%$, respectively, in the 40 and $25 \mathrm{HCC}$ specimens found to be positive for XAGE-1b and XAGE-1d mRNA expression by conventional RT-PCR. Compared to GAPDH expression, XAGE-1b and XAGE-1d mRNA copy numbers were 15.86 and $3.51 \%$, respectively, in testis tissues.

XAGE-1 isoform expression in HCC cancer by immunohistochemistry. XAGE-1 protein expression was examined in 96 HCC specimens by immunohistochemistry using a XAGE-1 $\mathrm{mAb}$ that reacted against XAGE-1 isoforms, including XAGE-1b, XAGE-1c and XAGE-1d. Cases with staining in $>10 \%$ of the cells were considered to be positive. Positive staining was observed in 39 of 40 (97.5\%) of the XAGE-1b mRNA-positive specimens, while no positive staining was noted in mRNA-negative specimens (Fig. 4). No positive staining was observed in the adjacent non-cancerous, relatively normal liver tissues examined.

Correlation between XAGE-1b and clinicopathological characteristics. The various clinicopathological characteristics of the patients and their tumors were compared according to the XAGE-1b mRNA expression (Table II). No significant association was found between age, gender, HBV infection, tumor size, AFP level, TNM stage or differentiation. The effect of XAGE-1b mRNA expression on survival was examined. Adequate clinical follow-up information was available for the 96 cases. The mean follow-up of the 96 cases was 12.8 months, (range 1-29). A total of 68 patients (70.8\%) survived, but 28 patients $(29.2 \%)$ succumbed during the follow-up period. The 2-year survival rate of the patients with a positive and negative XAGE-1b expression was 37.1 and $67.3 \%$, respectively, and the former group exhibited a relatively shorter 2-year survival rate $(\mathrm{P}=0.045)$. The Kaplan-Meier survival curves showed that patients with a positive XAGE-1b expression did not have a significant survival difference compared to patients with a negative XAGE-1b mRNA expression $(\mathrm{P}=0.182)$ (Fig. 5).

\section{Discussion}

The present investigation showed that XAGE-1 expression occurred in tumor samples from HCC patients, demonstrating a relatively high frequency expression of XAGE- $1 \mathrm{~b}$ and XAGE-1d (41.7 and 26.0\%, respectively). Additionally, the XAGE-1 protein was detected in 39 (40.6\%) HCC 
Table II. Correlation between XAGE-1b, the dominant type, XAGE-1c and XAGE-1d mRNA expression and clinicopathological characteristics in HCC.

\begin{tabular}{|c|c|c|c|c|c|c|c|}
\hline & $\begin{array}{l}\text { XAGE-1b- } \\
\text { positive }\end{array}$ & $\begin{array}{l}\text { XAGE-1b- } \\
\text { negative }\end{array}$ & P-value & One positive & Double positive & Triple positive & Tetra positive \\
\hline Cases & $40(41.7 \%)$ & $56(58.3 \%)$ & & 16 & 9 & 14 & 1 \\
\hline Age $($ mean \pm SD $)$ & $54.3 \pm 12.7$ & $51.3 \pm 11.1$ & 0.97 & 16 & 9 & 14 & 46 \\
\hline$\leq 50$ years & 18 & 25 & & 8 & 5 & 4 & 0 \\
\hline$>50$ years & 22 & 31 & & 8 & 4 & 10 & 1 \\
\hline Gender & & & 1.00 & & & & \\
\hline Male & 30 & 42 & & 9 & 9 & 11 & 1 \\
\hline Female & 10 & 14 & & 7 & 0 & 3 & 0 \\
\hline $\mathrm{HBV}$ & & & 0.53 & & & & \\
\hline Positive & 30 & 45 & & 13 & 7 & 10 & 0 \\
\hline Negative & 10 & 11 & & 3 & 2 & 4 & 1 \\
\hline AFP level & & & 0.29 & & & & \\
\hline$\leq 20 \mu \mathrm{g} / \mathrm{l}$ & 16 & 28 & & 8 & 2 & 5 & 1 \\
\hline$>20 \mu \mathrm{g} / 1$ & 24 & 27 & & 8 & 7 & 9 & 0 \\
\hline Tumor size & & & 0.70 & & & & \\
\hline$\leq 5 \mathrm{~cm}$ & 22 & 33 & & 10 & 2 & 9 & 1 \\
\hline$>5 \mathrm{~cm}$ & 18 & 23 & & 6 & 7 & 5 & 0 \\
\hline TNM stage & & & 0.88 & & & & \\
\hline $\mathrm{I} / \mathrm{II}$ & 27 & 37 & & 9 & 5 & 12 & 1 \\
\hline III/IV & 13 & 19 & & 7 & 4 & 2 & 0 \\
\hline Differentiation & & & 0.20 & & & & \\
\hline Well/moderate & 32 & 50 & & 15 & 6 & 10 & 1 \\
\hline Poor & 8 & 6 & & 1 & 3 & 4 & 0 \\
\hline
\end{tabular}

One positive, only XAGE-1b is positive; double positive, XAGE-1b and XAGE-1a or XAGE-1b and XAGE-1d are positive; triple positive, XAGE-1b, XAGE-1c and XAGE-1d are positive; tetra positive, XAGE-1b, XAGE-1a and XAGE-1c and XAGE-1d are positive. SD, standard deviation.

tumor tissues, rendering them ideal candidate antigens for antigen-specific HCC immunotherapy. Moreover, our study qualitatively showed that XAGE-1b and XAGE-1d exhibited a higher expression in certain HCC cases compared to testis tissues. Concomitantly, we found no relationship between the expression of XAGE-1b and the clinical parameters. Furthermore, the HCC patient groups with a positive XAGE-1 mRNA expression had a relatively shorter 2-year survival rate compared to the negative group.

Studies have shown that XAGE-1 was highly expressed in numerous malignancies. Using automated high-throughput filter immunoscreening or cDNA phage surface display, XAGE-1 antigen was identified in prostate cancer, particularly in the serum of prostate cancer and lung adenocarcinoma patients (17-19). The molecular mechanism regulating the expression of this CTA gene may be epigenetic modulation of the gene promoter, histone deacetylase and DNA methyltransferase inhibitors (20). Epigenetic modulation of this antigen gene may be found in tumorigenic human mesenchymal stem cells that can potentially be utilized in cancer therapy (21).

Furthermore, the dominant XAGE-1 isoform, XAGE-1b, is known to be able to stimulate the immune response of patients suffering from non-small cell lung or prostate cancer
(22-24). In addition, antibody responses to recombinant L552S protein, an alternatively spliced isoform of XAGE-1, were observed in the pleural effusion fluids of lung cancer patients (25). The XAGE-1b protein can be processed, is present in the HLA-I molecule and stimulates the autologous CD4 ${ }^{+}$ T-lymphocyte response $(26,27)$. Patients with lung adenocarcinoma expressing both XAGE-1b and HLA class I antigens were associated with prolonged survival time (28).

Full-length XAGE-1b protein-pulsed dendritic cells induce a specific cytotoxic T-lymphocyte response in vitro that suggests a potential role of immunotherapy in various types of cancer (29). Therefore, XAGE-1b is a type of highly and specifically expressed XAGE-1 antigen in tumors that can be used as targets for immunotherapy. However, XAGE-1b expression and its correlations with the clinicopathological characteristics have yet to be adequately analyzed in human HCC cancer tissues. To the best of our knowledge, this is the first study regarding the relationship between XAGE-1 expression and clinical parameters in a larger sample of patients with HCC. Of note is that this study did not demonstrate statistical significance of the survival difference on the basis of XAGE-1b expression, which has been shown to be of prognostic significance in lung cancer, as reported by Kikuchi et al 
A

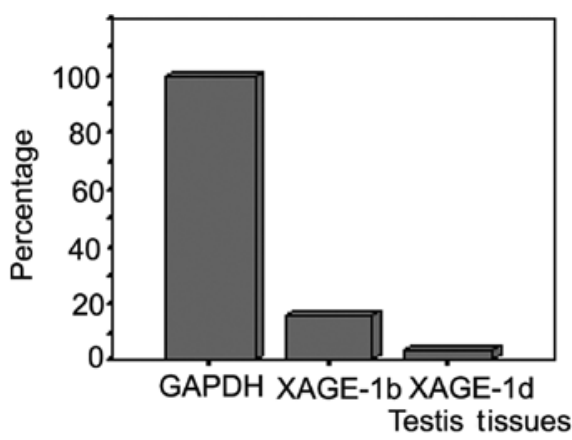

B

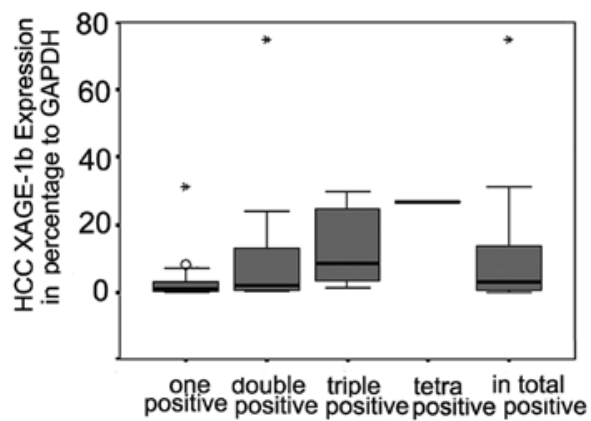

C

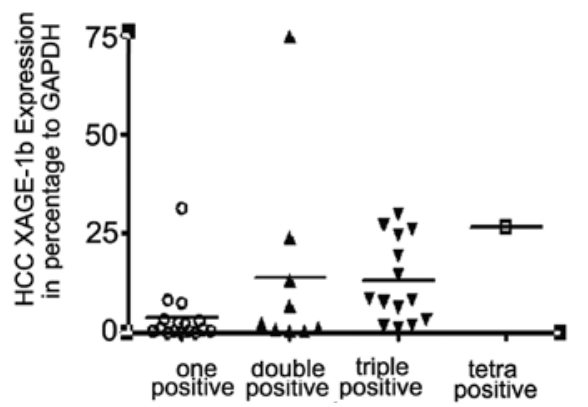

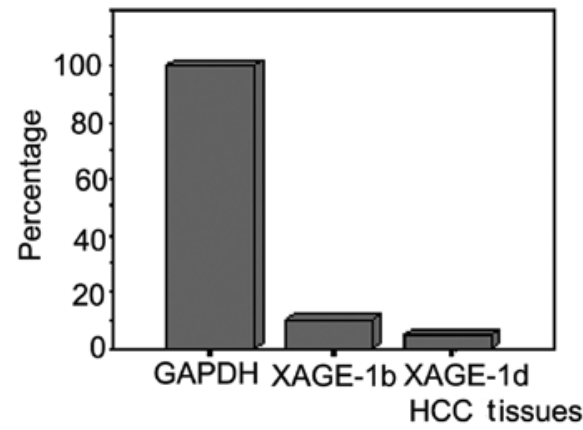
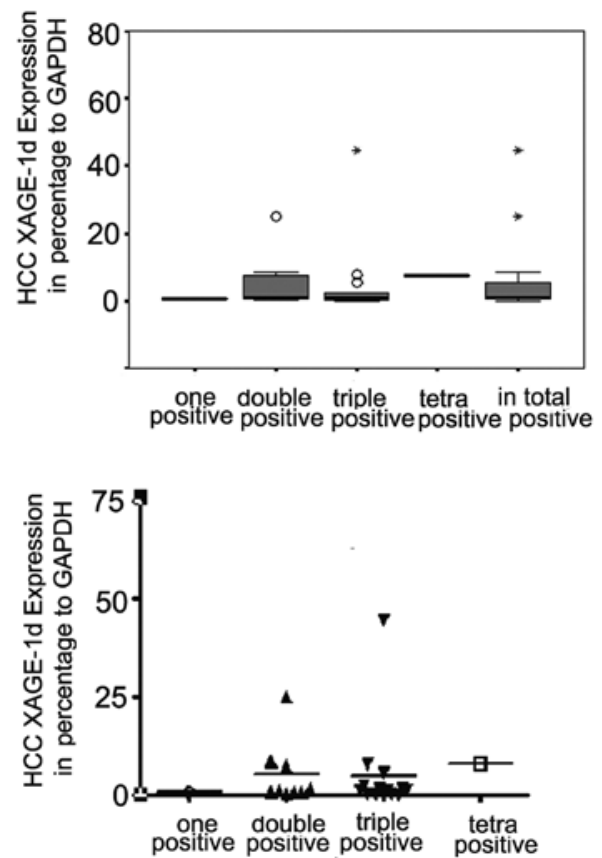

Figure 3. XAGE-1 mRNA expression in HCC and testis tissues. Histogram plots show i) the expression of the candidate internal control gene GAPDH as $100 \%$, and ii) XAGE-1b and XAGE-1d compared to GAPDH in HCC and testis tissues. (A) The box bars show XAGE-1b and XAGE-1d expression compared to GAPDH in HCC tissues. (B) The data are presented in five groups: One positive, only XAGE-1b is positive; double positive, XAGE-1b and XAGE-1a or XAGE-1b and XAGE-1d is positive; triple positive, XAGE-1b, XAGE-1c and XAGE-1d is positive; tetra positive, XAGE-1b, XAGE-1a and XAGE-1c and XAGE-1d are positive; total positive included the four above-mentioned groups. (C) The distribution of the relative expression values compared to the internal control gene GAPDH is shown, with the groups divided as above.

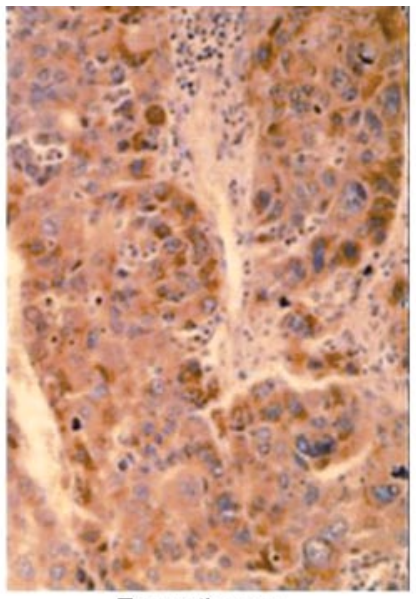

Tumor tissues

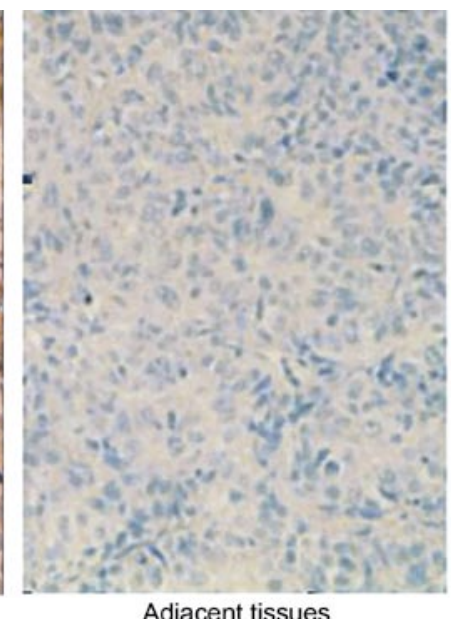

Adjacent tissues

Figure 4. Immunohistochemical staining of XAGE-1 protein in liver tissues. In the XAGE-1b mRNA-positive HCC tissues, the cancer tissue cells were randomly and heterogeneously reactive for XAGE-1 protein, while in the XAGE-lb mRNA-negative HCC tissues, the cancer tissue cells showed a negative expression of XAGE-1.

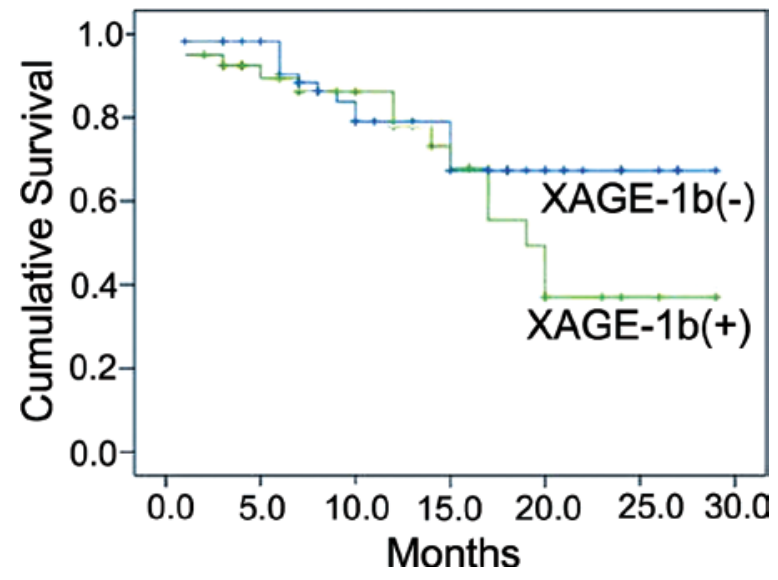

Figure 5. Overall survival curves following surgical therapy grouped by XAGE-1b expression and calculated using the Kaplan-Meier method. Statistical analysis showed that the positive XAGE-lb expression group $(\mathrm{n}=40$, green line) had no significant survival difference from the negative XAGE-1b expression group $(\mathrm{n}=56$, blue line $)(\mathrm{P}=0.182)$. 
(28). This result may be due to the fact that our study did not simultaneously evaluate the MHC-I expression status.

Further prospective studies with a larger number of cases in a wide range of clinical settings are required to evaluate XAGE-1b expression in order for the unique role of XAGE-1b in HCC development and progression to be determined. Moreover, humoral immunity and autologous T-lymphocyte responses induced by XAGE-1b should also be assayed. The results suggest that potential strategies to reduce or antagonise the XAGE-1b expression may become a valuable therapeutic approach in the treatment of HCC patients. Furthermore, due to its highly specific expression and immunogenic effects, XAGE-1b may be used as a vaccine applicable in the immunotherapy of HCC. However, further investigations regarding the application of XAGE-1b are crucial.

\section{References}

1. Bruix J, Boix L and Sala M: Focus on hepatocellular carcinoma. Cancer Cell 5: 215-219, 2004.

2. Tang ZY: Hepatocellular carcinoma - cause, treatment and metastasis. World J Gastroenterol 7: 445-454, 2001.

3. Kim W, Gores G, Benson JT, Therneau TM and Melton LJ III: Mortality and hospital utilization for hepatocellular carcinoma in the United States. Gastroenterology 129: 486-493, 2005.

4. Chen $\mathrm{CH}$, Chen GL, Lee HS and Huang GT: Expressions of cancer-testis antigens in human hepatocellular carcinomas. Cancer Lett 164: 189-195, 2001.

5. Chomez P, De Backer O and Bertrand M: An overview of the MAGE gene family with the identification of all human members of the family. Cancer Res 61: 5544-5551, 2001.

6. Luo G, Huang S and Xie X: Expression of cancer-testis genes in human hepatocellular carcinomas. Cancer Immunity 2: 11-21, 2002.

7. Peng JR, Chen HS and Mou DC: Expression of cancer/testis (CT) antigens in Chinese hepatocellular carcinoma and its correlation with clinical parameters. Cancer Lett 219: 223-232, 2005.

8. Tajima K, Obata Y and Tamaki H: Expression of cancer/testis (CT) antigens in lung cancer. Lung Cancer 42: 23-33, 2003.

9. Caballero OL and Chen YT: Cancer/testis (CT) antigens: potential targets for immunotherapy. Cancer Sci 100: 2014-2021, 2009.

10. Brinkmann U, Vasmatzis G and Lee B: Novel genes in the PAGE and GAGE family of tumor antigens found by homology walking in the dbEST database. Cancer Res 59: 1445-1448, 1999.

11. Liu XF, Helman LJ and Yeung C: XAGE-1, a new gene that is frequently expressed in Ewing's sarcoma. Cancer Res 60: 4752-4755, 2000.

12. Zendman AJ, van Kraats AA and den Hollander AI: Characterization of XAGE-1b, a short major transcript of cancer/testis-associated gene XAGE-1, induced in melanoma metastasis. Int J Cancer 97: 195-204, 2002.

13. Zendman AJ, van Kraats AA and Weidle UH: The XAGE family of cancer/testis-associated genes: alignment and expression profile in normal tissues, melanoma lesions and Ewing's sarcoma. Int J Cancer 99: 361-369, 2002.
14. Egland KA, Kumar V and Duray P: Characterization of overlapping XAGE-1 transcripts encoding a cancer testis antigen expressed in lung, breast, and other types of cancers. Mol Cancer Ther 1: 441-450, 2002.

15. Jun HL, Sang PK and Edward G: Activation of human cancer/ testis antigen gene, XAGE-1, in tumor cells is correlated with CpG island hypomethylation. Int J Cancer 116: 200-206, 2005.

16. Sato S, Noguchi Y and Ohara N: Identification of XAGE-1 isoforms: predominant expression of XAGE-1b in testis and tumors. Cancer Immunity 7: 5-12, 2007.

17. Alsoe L, Stacy JE and Fossa A: Identification of prostate cancer antigens by automated high-throughput filter immunoscreening. J Immunol Methods 330: 12-23, 2008.

18. Fossa A, Alsoe L and Crameri R: Serological cloning of cancer/ testis antigens expressed in prostate cancer using cDNA phage surface display. Cancer Immunol Immunother 53: 431-438, 2004.

19. Ali Eldib AM, Ono T and Shimono M: Immunoscreening of a cDNA library from a lung cancer cell line using autologous patient serum: identification of XAGE-1b as a dominant antigen and its immunogenicity in lung adenocarcinoma. Int $\mathrm{J}$ Cancer 108: 558-563, 2004.

20. James SR, Link PA and Karpf AR: Epigenetic regulation of X-linked cancer/germline antigen genes by DNMT1 and DNMT3b. Oncogene 25: 6975-6985, 2006.

21. Gjerstorff M, Burns JS and Nielsen O: Epigenetic modulation of cancer-germline antigen gene expression in tumorigenic human mesenchymal stem cells: implications for cancer therapy. Am J Pathol 175: 314-323, 2009.

22. Koizumi F, Noguchi Y and Saika T: XAGE-1 mRNA expression in prostate cancer and antibody response in patients. Microbiol Immunol 49: 471-476, 2005.

23. Nakagawa K, Noguchi Y and Uenaka A: XAGE-1 expression in non-small cell lung cancer and antibody response in patients. Clin Cancer Res 11: 5496-5503, 2005.

24. Watanabe $Y$ and LePage S: Characterization of preexisting humoral immunity specific for two cancer-testis antigens overexpressed at the mRNA level in non-small cell lung cancer. Cancer Immunity 6: 3-11, 2006.

25. Wang T, Fan L and Watanabe Y: L552S, an alternatively spliced isoform of XAGE-1, is overexpressed in lung adenocarcinoma. Oncogene 20: 7699-7709, 2001.

26. Morishita Y, Uenaka A and Kaya S: HLA-DRB1*0410-restricted recognition of XAGE-1b 37-48 peptide by CD4 T cells. Microbiol Immunol 51: 755-762, 2007.

27. Shimono M, Uenaka A and Noguchi Y: Identification of DR9-restricted XAGE antigen on lung adenocarcinoma recognized by autologous CD4 T-cells. Int J Oncol 30: 835-840, 2007.

28. Kikuchi E, Yamazaki K and Nakayama E: Prolonged survival of patients with lung adenocarcinoma expressing XAGE-1b and HLA class I antigens. Cancer Immunity 8: 13-18, 2008.

29. Zhou Q, Guo AL and Xu CR: A dendritic cell-based tumour vaccine for lung cancer: full-length XAGE-1b protein-pulsed dendritic cells induce specific cytotoxic $\mathrm{T}$ lymphocytes in vitro. Clin Exp Immunol 153: 392-400, 2008. 